\title{
Visualizing the Histotripsy Process: Bubble Cloud-Cancer Cell Interactions in a Tissue-Mimicking Environment
}

Eli Vlaisavljevich ${ }^{1}$, Adam Maxwell ${ }^{2}$, Lauren Mancia ${ }^{3}$, Eric Johnsen ${ }^{3}$, Charles Cain ${ }^{1}$, Zhen Xu ${ }^{1,4}$

1 - Department of Biomedical Engineering, University of Michigan, Ann Arbor, MI

2 - Department of Urology, University of Washington, Seattle, WA

3 - Department of Mechanical Engineering, University of Michigan, Ann Arbor, MI

4 - Department of Pediatrics and Communicable Diseases, Division of Pediatric Cardiology, University of Michigan, Ann Arbor, MI

\section{Corresponding Author: Eli Vlaisavljevich}

Eli Vlaisavljevich,

University of Michigan

Department of Biomedical Engineering

2200 Bonisteel Blvd, Ann Arbor, MI 48109, USA.

Phone: (734) 936-3674

E-mail: evlaisav@umich.edu 
Abstract-Histotripsy is a non-invasive ultrasonic ablation method that uses cavitation to mechanically fractionate tissue into acellular debris. With a sufficient number of pulses, histotripsy can completely fractionate tissue into a liquid-appearing homogenate with no cellular structures. The location, shape, and size of lesion formation closely match those of the cavitation cloud. Previous work has led to the hypothesis that the rapid expansion and collapse of histotripsy bubbles fractionate tissue by inducing large stress and strain on the tissue structures immediately adjacent to the bubbles. In this work, the histotripsy bulk tissue fractionation process is visualized at the cellular level for the first time using a custom-built $2 \mathrm{MHz}$ transducer incorporated into a microscope stage. A layer of breast cancer cells were cultured within an optically transparent fibrin-based gel phantom to mimic cells inside a three dimensional extracellular matrix. To test the hypothesis, the cellular response to single and multiple histotripsy pulses was investigated using high speed optical imaging. Bubbles were always generated in the extracellular space, and significant cell displacement/deformation was observed for cells directly adjacent to the bubble during both bubble expansion and collapse. The largest displacements were observed during collapse for cells immediately adjacent to the bubble, with cells moving more than 150$300 \mu \mathrm{m}$ in less than $100 \mu \mathrm{s}$. Cells often underwent multiple large deformations (>150\% strain) over multiple pulses, resulting in the bisection of cells multiple times before complete rupture. To provide theoretical support to the experimental observations, a numerical simulation was conducted using a single bubble model, which showed that histotripsy exerts the largest strains and cell displacements in the regions immediately adjacent to the bubble. The experimental and simulation results support our hypothesis, which helps to explain the formation of the sharp lesions formed in histotripsy therapy localized to the regions directly exposed to the bubbles.

Keywords: Histotripsy, Cavitation, Cancer, Cell-bubble interactions, Tissue fractionation 


\section{Introduction}

Histotripsy is a non-invasive ablation method that controls cavitation to mechanically fractionate soft tissue through high pressure ( $>10 \mathrm{MPa})$, short duration $(<20 \mu \mathrm{sec})$ ultrasound pulses at low duty cycles $(<1 \%$ ) (Xu et al. 2005a; Maxwell et al. 2011; Maxwell et al. 2013; Vlaisavljevich et al. 2014b; Vlaisavljevich et al. 2015a). Histotripsy depends on the initiation and maintenance of a cavitation bubble cloud to produce mechanical tissue fractionation $(\mathrm{Xu}$ et al. 2005b; Parsons et al. 2006a). The cavitation bubble cloud can produce mechanical tissue damage by generating a bubble cloud at a fluid-tissue interface (i.e. tissue erosion) (Xu et al. 2010; Owens et al. 2011) or by generating a bubble cloud inside of a bulk tissue (i.e. tissue fractionation) (Roberts et al. 2006; Hall et al. 2007; Hempel et al. 2011; Vlaisavljevich et al. 2013b). For bulk tissue fractionation, histotripsy can completely fractionate the tissue into a liquid-appearing homogenate with no cellular structures remaining (Roberts et al. 2006; Hall et al. 2007; Hempel et al. 2011; Vlaisavljevich et al. 2013b). Previous work has shown that histotripsy can produce precise lesions, with bisected cells often observed at the sharply demarcated lesion boundary (Parsons et al. 2006a; Roberts et al. 2006; Hall et al. 2007; Hempel et al. 2011; Vlaisavljevich et al. 2013b).

Some effort has been made towards understanding the histotripsy process and the potential mechanisms with which the cavitation bubbles induce tissue damage. Numerous studies have investigated the interaction of cells with cavitating microbubbles both theoretically and experimentally, with results showing several effects of cavitation that may contribute to tissue damage including bubble expansion, bubble collapse, translational motion of bubbles against a

tissue interface, shockwave emission, and micro-jetting (Vogel et al. 1996; Cochran and Prausnitz 2001; Sundaram et al. 2003; Brujan 2004; Kimmel 2006; Ohl et al. 2006; Wu 2007; 
Sankin et al. 2010; Chen et al. 2011; Quinto-Su et al. 2011; Fan et al. 2012; Li et al. 2013; Yuan et al. 2015). However, these prior studies have been almost exclusively focused on isolated cells inside a fluid environment or at a fluid-cell interface. While the results of these studies provide some insight into the mechanism with which cavitation bubbles may induce tissue damage at a fluid interface in histotripsy erosion, they provide only limited insight into the process of cell damage caused during histotripsy bulk tissue fractionation, which occurs within a three dimensional extracellular matrix. Therefore, the methods used in this work aim to investigate the histotripsy bulk tissue fractionation process at the cellular level in a relevant tissue mimicking environment.

In order to understand the histotripsy bulk tissue fractionation process, previous studies in our lab have utilized a cavitation-indicating tissue phantom to visualize the cavitation cloud and the lesion formation (Maxwell et al. 2010; Vlaisavljevich et al. 2013a; Lin et al. 2014). This tissue phantom consists of transparent agarose gel with a layer of red blood cells (RBCs) that turns from opaque to translucent after being fractionated (Maxwell et al. 2010; Vlaisavljevich et al. 2013a; Lin et al. 2014). High speed images of histotripsy treatments of RBC phantoms have shown that the location, shape, and size of the lesion closely match those of the cavitation cloud. Furthermore, single bubbles on the cloud periphery have been observed to cause localized damage similar in size to the maximum expanded diameter of individual bubbles. These studies have led to the hypothesis that the rapid expansion and collapse of histotripsy bubbles fractionate tissue by inducing large stress and strain on the adjacent tissue structures. However, the process of histotripsy tissue fractionation has not yet been visualized on a cellular level or on the time scales at which the damage is achieved. Additionally, while RBC phantoms are a good model to visualize the area ablated by histotripsy, the model is limited to suspended RBCs that fractionate 
at significantly smaller deformations than adherent cells attached to an extracellular matrix (ECM) (Barbee 2005; Hammond and Kamm 2008; Kilinc et al. 2009; Ofek et al. 2010). Therefore, the question remains as to how histotripsy fractionates adherent cells within an ECM that can undergo much larger deformations before rupture (Barbee 2005; Hammond and Kamm 2008; Kilinc et al. 2009; Ofek et al. 2010).

In this study, the process by which cells are fractionated and completely ruptured by histotripsy was visualized at the cellular level for the first time. Ultrasound pulses were generated using a custom-built transducer incorporated into the stage of an inverted microscope, which was coupled to high-speed imaging. Optically transparent tissue phantoms with cancer cells embedded inside fibrin-based gels were used to mimic real tissue with cells adherent to a three dimensional matrix, and the bubble-cell interactions produced by a single histotripsy pulse as well as over multiple histotripsy pulses were imaged with a high-speed camera. We hypothesize that each histotripsy pulse produces cavitation bubbles in the ECM outside the cells, and the expansion and collapse of the bubbles exerts large transient strains on the surrounding tissue structures to cause fractionation of cells immediately adjacent to the cavitation bubbles. Cells that are not immediately adjacent to the cavitation bubbles also experience transient strain, but the strain is not sufficiently high to result in fractionation. Over multiple pulses, all of the cells inside the focal region containing the bubble cloud are completely fractionated while the cells in the surrounding tissue remain intact, resulting in the localized histotripsy-induced tissue damage observed in previous studies. To test our hypothesis, the cell displacements/deformations observed from high-speed images were compared with the timing of bubble expansion and collapse. Cell displacement/deformation was also compared as a function of the distance away from the bubble. In the final part of this study, the strain induced to the surrounding media 
during the expansion and collapse of a histotripsy bubble was investigated theoretically using a single bubble numerical model, with the results compared to the experimental findings.

\section{Methods}

Miniature Transducer-tank Setup

A custom-built miniature transducer tank (Fig.1A) was designed to generate and image a histotripsy bubble cloud-cell interaction using an inverted microscope (Nikon Eclipse Ti, 10X magnification), which was coupled to a high-speed camera. The miniature transducer-tank was designed to achieve short, high-pressure histotripsy pulses and fit into a 16x11 cm microscope stage with an optical window at the bottom of tank for the microscope objective. Four, 2-cm diameter piezoceramic elements (Elements; Steiner \& Martins) were positioned $3 \mathrm{~cm}$ apart on the semicircular side of the tank wall. The transducer had center frequency of $2 \mathrm{MHz}$ and geometric focal length of $3 \mathrm{~cm}$. The transducer tank was filled with degassed water at room temperature $\left(\sim 20^{\circ} \mathrm{C}\right)$ with elements completely submerged. A two axis micro-positioning system was built support the tissue phantom and to adjust the position of the phantom with respect to the transducer focus.

\section{Ultrasound Generation}

Histotripsy pulses were generated by the 4 element, $2 \mathrm{MHz}$ microscope transducer. Input signals were provided by a custom built field-programmable gate array (FPGA) board (Altera Corporation, San Jose, CA, USA) that functioned as a signal generator. The transducer was

calibrated using a fiber optic probe hydrophone built in house (Parsons et al. 2006b) and the 
focal pressure waveforms were recorded in free-field in degassed water at $20^{\circ} \mathrm{C}(\mathbf{F i g . 1 B})$. Bubble clouds were produced with peak negative pressure of $\sim 11 \mathrm{MPa}$ and a pulse length of 10 cycles.

\section{Cancer Cell Tissue Phantom Preparation}

Since previous work has demonstrated that tissue mechanical properties significantly impact histotripsy (Xu and Bigelow 2011; Vlaisavljevich et al. 2014a; Vlaisavljevich et al. 2014b; Xu et al. 2014; Vlaisavljevich et al. 2015a; Vlaisavljevich et al. 2015b), an optically transparent fibrin-based tissue phantom with an embedded layer of human breast cancer cells (BCCs, MDAMB231) was used to mimic adherent cells inside a tissue-like ECM environment. Cell culture work was performed under ThermoForma Class II A1 Biological Safety Cabinet and cells were incubated at $37^{\circ} \mathrm{C}$ and $5 \% \mathrm{CO}_{2}$. BCCs were cultured in Dulbecco's Modified Eagle's Medium (DMEM; Gibco) supplemented with 10\% fetal bovine serum (FBS; Media Tech) and $1 \%$ penicillin/streptomycin (P/S; Media Tech). BCCs were passaged every six days with media change every third day. Cell washes were performed with sterile phosphate buffered saline (PBS; Gibco). BCCs were detached for experimental seeding with $0.05 \%$ Trypsin/0.53mM EDTA in HBSS without sodium bicarbonate, calcium, and magnesium (Trypsin-EDTA; Cellgro). BCCs were used prior to passage 15 and were seeded $\left(2 \times 10^{4}\right.$ cells/ $\left./ \mathrm{cm}^{2}\right)$ onto a fibrin gel inside cell culture boxes made of acoustically transparent, $0.1 \mathrm{~mm}$ thick polycarbonate (Polycarbonate; McMaster). Cell boxes (20mm x $20 \mathrm{~mm} \times 40 \mathrm{~mm})$ were washed 3 times in $95 \%$ ethanol and sterilized 20 minutes under ultra violet light prior to use. Cells were seeded onto $4 \mathrm{~mm}$ thick fibrin gel to mimic extracellular matrix within tissue (fibrin gel was chosen for similar acoustic and mechanical properties to soft tissue). To make the fibrin gel, a fibrinogen solution (Fibrinogen from bovine plasma; Sigma Aldrich) of $10 \mathrm{mg} / \mathrm{ml}$ was prepared in the culture 
medium and split into cell boxes. Thrombin (Thrombin; Sigma Aldrich) was then added (20 $\mu \mathrm{l} / \mathrm{ml}$ ) along with $5 \% \mathrm{FBS}$, and the solution was incubated at $37^{\circ} \mathrm{C}$ for 1 hour prior to seeding to ensure solidification of fibrin. The shear modulus of the fibrin gel $(10 \mathrm{mg} / \mathrm{ml})$ used in this study has previously been measured to be $\sim 377 \mathrm{~Pa}$ (Kotlarchyk et al. 2011). BCCs were cultured on fibrin for three days before histotripsy treatment. An additional $8 \mathrm{~mm}$ thick fibrin gel was added over cells 1 day prior to treatment to mimic behavior inside a tissue ECM environment. During experiments, the cell monolayer was aligned with the axial plane of the transducer.

\section{Single Pulse Cell-bubble Interaction}

To study the bubble-cell interactions induced by a single histotripsy pulse, an ultra-fast digital camera (SIM 802, Optronis) was aligned with the inverted microscope left optical port. The camera was externally triggered to synchronize recorded images with the histotripsy pulse at specific delay intervals. A flash lamp was also triggered and positioned over cells to illuminate images with an exposure time of $2 \mu \mathrm{s}$. A single 10-cycle histotripsy pulse was applied to tissue phantoms containing an embedded BCC monolayer, and the camera was triggered to record 16 images with the first image taken $20 \mu \mathrm{s}$ before the pulse and 15 images taken after the arrival of the pulse with inter-frame durations between 4-6 $\mu$ s. Recorded images spanned the entirety of expansion and collapse of a bubble cloud after arrival of a single pulse and the subsequent cell response following collapse. Since images during the pulse were blurred due to the ultrasound wave interfering with imaging, the cell response during bubble expansion and collapse after the pulse was investigated. To quantify the cell displacements induced by a single histotripsy pulse, particle tracking analysis was used to measure the cell displacements for the cells surrounding 
the histotripsy bubbles. The cell displacements occurring during bubble collapse were plotted as a function of the distance from the bubble wall, as measured at the point of maximum expansion.

\section{Multiple Pulse Cell-bubble Interaction}

To study bubble-cell interactions over multiple pulses, a high-speed, 1 megapixel CCD camera (Phantom V210, Vision Research) with frame rate of 2000 fps was aligned to the inverted microscope left optical port as it allows up to 4000 image storage for each acquisition in comparison to the 16 frame storage for the ultra-fast SIM camera. The camera was externally triggered to synchronize recorded images with the histotripsy pulse at specific delay intervals. An exposure time of $20 \mu$ s was used for each recorded frame. 1000 histotripsy pulses (10-cycles, $50 \mathrm{~Hz}$ PRF) were applied to tissue phantoms containing an embedded BCC monolayer, and the camera was triggered to record images $24 \mu \mathrm{s}$ after the arrival of each pulse. The cellular response to histotripsy was analyzed after each pulse, and the number of cells in the focal region was compared as a function of pulse number. Videos were also analyzed to compare the location of the bubble cloud with the regions in which of cell rupture/removal were observed. In addition, although only a single image was captured after each pulse, the approximate cell deformation induced by histotripsy was estimated for cells surrounding the histotripsy bubbles by comparing the percent change in length of a cell after a given pulse compared to the initial length of the cell measured before histotripsy was applied.

\section{Histotripsy-induced Stress-Strain Simulation}

To investigate the stress and strain induced to tissue structures during the expansion and collapse of a histotripsy microbubble, a numerical model based on a Kelvin-Voigt viscoelastic 
model was used. Based on previous work suggesting that histotripsy tissue fractionation is limited to the regions directly exposed to the cavitation bubbles, we hypothesize that the histotripsy bubble will exert large strains on the surrounding media during both expansion and collapse, with the largest strains occurring in the regions immediately adjacent to the bubble. To test this hypothesis and provide theoretical explanation to the experimental observations, simulations exposed a $2.5 \mathrm{~nm}$ initial gas nucleus to a single peak negative pressure

$$
p_{a}(t)= \begin{cases}p_{A}\left(\frac{1+\cos [\omega(t-\delta)]}{2}\right)^{n}, & |t-\delta| \leq \frac{\pi}{\omega} \\ 0, & |t-\delta|>\frac{\pi}{\omega}\end{cases}
$$

where $\mathrm{p}_{\mathrm{A}}$ is the peak negative pressure, $\omega$ is the angular frequency of the ultrasound wave, $\delta$ is a time delay, and $n$ is a curve-fitting parameter set to $n=3.7$. These conditions were chosen based on previous work showing close agreement between the expansion and collapse behavior of simulated bubbles compared to experimentally observed histotripsy bubbles (Vlaisavljevich et al. 2015a; Vlaisavljevich et al. 2015b). For this study, we assume the surrounding medium to have homogeneous properties, and that the bubble contains air and remains spherical. Spherical bubble dynamics in general viscoelastic media were simulated using an in-house code (Warnez and Johnsen 2015). To describe the bubble response to a far-field pressure variation, $\mathrm{p}_{\text {inf }}(\mathrm{t})$, we solve the the compressible Keller-Miksis equation

$$
\left(1-\frac{\dot{R}}{c}\right) R \ddot{R}+\frac{3}{2}\left(1-\frac{\dot{R}}{3 c}\right) \dot{R}^{2}=\left(1+\frac{\dot{R}}{c}\right)\left[\frac{p_{B}-p_{\infty}(t)}{\rho}-\frac{4 \mu \dot{R}}{R}-\frac{2 S}{\rho R}-E_{N T}\right]+\frac{R}{\rho c} \frac{d}{d t}\left(p_{a}-p_{\infty}\right)
$$

where $\rho$ is the surrounding medium's density, $c$ is the speed of sound, $S$ is the surface tension, and $\mu$ is the viscosity. Here, the overdots $\left({ }^{*}\right)$ denote derivatives with respect to time, $t$. For simplicity, the air within the bubble behaves adiabatically, so that

$$
p_{g}=p_{0}\left(\frac{R_{0}}{R}\right)^{3 \kappa}
$$


where $\kappa=1.4$ is the ratio of specific heats for air, $R_{0}$ is the initial bubble radius, with

$$
p_{0}=p_{\infty}(0)+\frac{2 S}{R_{0}}
$$

The viscoelasticity of the medium is modeled using a Kelvin-Voigt approach, in which the stress consists of the sum of viscous and elastic contributions. Because of the large deformations during growth, a hyperelastic (Neo-Hookean) constitutive relation is used (Gaudron et al. 2015), to yield the following elastic term, $E_{N T}$,

$$
E_{N T}=\frac{G}{2}\left(5-4\left(\frac{R_{0}}{R}\right)-\left(\frac{R_{0}}{R}\right)^{4}\right)
$$

where $\mathrm{G}$ is the linear shear modulus. The equations are marched forward in time using explicit Runge-Kutta time marching with adaptive time stepping to compute the radius (and all relevant quantities) as a function of time. The properties $\rho=1000 \mathrm{~kg} / \mathrm{m}^{3}, c=1497 \mathrm{~m} / \mathrm{s}^{2}, \mu=$ $1.00 \mathrm{mPa} \cdot \mathrm{s}$ (viscosity of water), and $S=72 \mathrm{mN}-\mathrm{m}$ (water-air surface tension) were used to approximate the surrounding medium. The shear modulus was set to $\mathrm{G}=377 \mathrm{~Pa}$ to match the shear modulus of the fibrin tissue phantoms and the ultrasound frequency was set to $2 \mathrm{MHz}$ to match experiments.

To calculate the local finite strain fields in the medium surrounding the bubble, we use the logarithmic or Hencky strain measure (Holzapfel 2000):

$$
\mathbf{E}=\frac{1}{2} \ln \mathbf{C}
$$

where $\mathbf{E}$ is the finite strain tensor and $\mathbf{C}=\mathbf{F}^{\mathbf{T}} \mathbf{F}$ is the right Cauchy-Green deformation tensor. The deformation gradient tensor, $\mathbf{F}$ as defined in previous studies (Gaudron et al. 2015; Warnez and Johnsen 2015) is used to relate the initial, un-deformed configuration of the medium to its current, time-dependent configuration. This allows us to compute the radial and polar strain fields induced by the bubble in the medium: 


$$
\begin{gathered}
E_{r r}=\frac{2}{3} \ln \left(1-\frac{R^{3}-R_{0}^{3}}{r^{3}}\right) \\
E_{\theta \theta}=-\frac{1}{3} \ln \left(1-\frac{R^{3}-R_{0}^{3}}{r^{3}}\right)
\end{gathered}
$$

In the above strain expressions, $r$ denotes a position in the medium, $R$ is the position of the bubble wall calculated using E2-E5, and $R_{0}$ is the initial bubble radius. We can also calculate the strain on a particle or cell displaced with the medium as the medium is deformed by the bubble. The trajectory, $r(t)$ of a cell starting a given distance $r^{*}$ from the bubble nucleus is given by:

$$
r(t)=\sqrt[3]{\left(r^{*}\right)^{3}-R_{0}^{3}+R^{3}}
$$

Using this expression, we can calculate radial and polar strains on the cell at each time step along its trajectory. Figure 2 shows a schematic of the radial and polar strains applied to the media during bubble expansion and collapse. The bubble radius was graphed as a function of time, and the magnitudes of the strain fields were calculated for the region surrounding the bubble during both bubble expansion and collapse. In addition, the maximum strain applied to the media by the bubble was plotted as a function of distance from the bubble wall. All numerical simulations were conducted using a single bubble model and did not account for potential bubble-bubble interactions that may occur within a histotripsy bubble cloud.

\section{Results}

\section{Single Pulse Cell-bubble Interaction}

In the first part of this study, the bubble-cell interactions induced by a single histotripsy

pulse were investigated. Experiments exposing tissue phantoms containing a layer of embedded 
breast cancer cells to a single histotripsy pulse demonstrated that the histotripsy bubbles were generated in the regions between cells and induced significant cell displacement and deformation to the surrounding cells. Figure 3 shows a representative video of the cellular response to a single histotripsy pulse. For all experiments, histotripsy bubbles were generated in the regions outside of the cell (i.e. inside the ECM), with no observed instances of bubbles being generated and expanded inside of the cells.

Comparing the response of cells exposed to the histotripsy bubbles demonstrated that the bubbles induced transient strains to the surrounding cellular structures. Significant cell displacement was observed during both expansion and collapse, as well as after bubble collapse due to the tissue inertial response. For example, the cells shown in Figure $\mathbf{3}$ were displaced in the direction of the radial bubble wall during expansion (first five frames), with the rate of cell displacement decreasing as the bubble reached its maximum size (Fig.3). During collapse, larger cell displacements were observed, with the cells immediately surrounding the bubble translated toward the collapsing bubble (Fig.3). After collapse, the cells that remained continued to be displaced due to the tissue inertial response before returning to their initial positions $<100 \mu \mathrm{s}$ after the pulse arrived at the focus, demonstrating the transient nature of the applied strain. For cells directly exposed to the bubbles, significantly fewer cells remained after bubble collapse, with these cells irreversibly ruptured or detached from the fibrin matrix (Fig.3).

Further analysis of the cellular response demonstrated that the largest cell displacements were observed for cells immediately adjacent to the bubble wall during collapse. For example, cells directly adjacent to the large bubble shown in Figure 3 moved more than $200 \mu \mathrm{m}$ during bubble collapse, with half of the cells exposed to the bubble (top right corner) remaining after the treatment. While the cells immediately adjacent to the bubble experienced displacements of 
hundreds of microns, smaller displacements (i.e. $<50 \mu \mathrm{m}$ ) were observed for cells further away from the bubble, with these cells returning to their initial positions and remaining intact after the treatment. The displacements of the cells during bubble collapse were analyzed as a function of distance from the bubble wall, with results demonstrating a significant decrease in cell displacement with increasing distance from the bubble following an inverse power relationship (Fig.4). The cell displacements ranged from $\sim 150-350 \mu \mathrm{m}$ for cells within $25 \mu \mathrm{m}$ of the bubble wall to displacements of $<50 \mu \mathrm{m}$ for cells $>250 \mu \mathrm{m}$ from the bubble wall (Fig.4). Power regression analysis demonstrated this trend was statistically significant, with an $R^{2}$ value of 0.74 .

\section{Multiple Pulse Cell-bubble Interactions}

In the second part of this study, the histotripsy fractionation process occurring over multiple pulses was investigated. Results demonstrated that histotripsy significantly deformed and completely fractionated all of the cells exposed to the bubble cloud at the focus while the cells in the surrounding regions experienced smaller deformations and remained intact after treatment. Over multiple pulses, cell rupture/bisection and complete removal occurred for all cells within the region exposed to the bubble cloud, resulting in the formation of a histotripsy lesion with sharp boundaries between the completely fractionated acellular focal region and the surrounding region containing intact cells (Fig.5). As the tissue fractionation progressed, cells (or parts of cells) were observed to be pulled towards the bubble cloud, similar to what was observed in the single pulse experiments after bubble collapse. Many of the cells in the focal region experienced multiple large deformations (i.e. cells stretching to $>150 \%$ of original length) prior to rupture. A significant increase in both bubble cloud size and lesion area was observed with increasing number of pulses, with a clearly defined lesion containing no cells remaining 
after 1000 pulses. This lesion was characterized by a sharply demarcated boundary between the focal region which contained no remaining cells and the surrounding region in which the cells remained intact. At this boundary, some bisected cells were observed after treatment, similar to previous histological observations.

Analysis of the multiple pulse experiments demonstrated that cells at the boundary of the expanding histotripsy lesion remained intact for many pulses before complete removal, with cells (or parts of cells) at the lesion boundary were pulled towards the bubble cloud (Fig.5). Cells at the lesion boundary underwent multiple large strains and were often bisected multiple times before complete removal (Fig.6). For example, the cell indicated by the arrow in Figure 6 was pulled towards the bubble cloud and underwent at least three significant deformations ( $\sim 150 \%$ $250 \%$ of original length) over approximately 40 pulses, resulting in the cell being bisected twice before complete removal. Since only one time point was visualized per pulse, it is possible that the cell underwent additional deformations during this period that were not directly observed. Although the boundary cells were deformed after each pulse, cell bisection was only observed on pulses when very large deformations (i.e. cellular strains $>0.5 \mu \mathrm{m} / \mu \mathrm{m}$ ) were induced. As shown in the video in Figure 5, these large deformations and cell bisections were only induced to the boundary cells on pulses in which a bubble formed directly adjacent to the cell, while significantly smaller deformations (and no cell rupture) were observed on pulses in which the bubbles formed further away (i.e. $>\sim 25-50 \mu \mathrm{m}$ ) from the cell. This finding closely matched the results from the single pulse experiments which demonstrated a significant increase in cell displacement for cells closer to the histotripsy bubble. It is also worth noting that the treated region became more fluid-like over the course of treatment, suggesting that the bubble-cell interactions at the lesion boundary at later time points may be similar to bubble-cell interactions 
at a fluid-tissue interface, once the tissue has been fractionated to a certain point. This possibility would be consistent with the results showing cells (or parts of cells) at the lesion boundary pulled towards the bubble cloud, which is similar to what was observed in the single pulse experiments after bubble collapse as well the results of previous studies showing blood vessel invaginations after bubble collapse inside of the vessel (Chen et al. 2011).

Results from the multiple pulse experiments also demonstrated that, rather than killing isolated cells, histotripsy tissue fractionation involves the incremental breakdown of an interconnected cell/matrix structure. Similar to the single pulse experiments, histotripsy bubbles were observed to originate in the regions between cells (i.e. inside the ECM), with the bubbles exerting transient strains on the surrounding media consisting of both cells and the fibrin matrix. Over the course of multiple pulses, cells inside the focal volume were either detached from the matrix (rupture of the cell-matrix adhesions) or bisected (i.e. rupture of the cells). After cell bisection, the remaining portions of the cell continued to be deformed, demonstrating the interconnected nature of the intracellular and extracellular matrix. In some cases, cell deformation appeared to be induced directly from bubble-cell interactions. However, smaller cell deformation and displacement was also observed for cells further from the bubble, likely due to the strain induced by the bubbles on the ECM, similar to what was observed in the single pulse experiments.

\section{Histotripsy Bubble-induced Strain Simulation}

The strain induced to tissue structures during the expansion and collapse of a histotripsy microbubble were investigated theoretically using a single bubble numerical model as described above. Result supported the hypothesis that histotripsy bubbles induce large transient strains on 
the adjacent tissue structures during both expansion and collapse, with the largest strains induced in the regions directly exposed to the bubble (Fig.7). Following the passage of the pulse, the bubble rapidly expanded, slowing down as it reached a maximum radius $\left(R_{\max }\right)$, and then collapsed violently. The strain applied to the media increased as the bubble expanded, with the maximum radial strain $\left(E_{r r_{-} \max }\right)$ and the maximum polar strain $\left(E_{\theta \theta_{-} \max }\right)$ occurring at $R_{\max }$. For example, a simulation of a histotripsy bubble which expanded to an $R_{\max } \sim 200 \mu \mathrm{m}$ demonstrated large radial strains (Fig.7A) and polar strains (Fig.7B) were induced at the bubble wall during both the expansion and collapse phases, with the largest strains occurring at $R_{\max }$.

Comparing the maximum strain induced in the media as a function of distance from a bubble demonstrated a decrease in the maximum strain with increasing distance from the initial bubble following an inverse power relationship (Fig.8). For example, $E_{r r_{-} \max }$ decreased from 10.6 $\mu \mathrm{m} / \mu \mathrm{m}$ for a particle $1 \mu \mathrm{m}$ from the initial bubble nuclei to $0.04 \mu \mathrm{m} / \mu \mathrm{m}$ for a particle $500 \mu \mathrm{m}$ from the initial bubble nuclei (Fig.8A). Similarly, $E_{\theta \theta_{-} \max }$ decreased from $5.3 \mu \mathrm{m} / \mu \mathrm{m}$ for a particle $1 \mu \mathrm{m}$ from the initial bubble nuclei to $0.02 \mu \mathrm{m} / \mu \mathrm{m}$ for a particle $500 \mu \mathrm{m}$ from the initial bubble nuclei (Fig.8B). In addition to the decrease in strain with increasing distance from the bubble, a similar decrease in cell displacement was observed with increasing distance from the bubble. For example, the black lines in Figure 7 show the trajectories of particles starting at various distances from the bubble nucleus and moving with the medium as it is deformed. The particle displacement results demonstrated significant decrease in displacement with increasing distance from the bubble. These results closely matched the experimental results from the single bubble experiments which also demonstrated a decrease in cell displacement with increasing distance from the bubble following an inverse power relationship. 


\section{Discussion}

In this study, the histotripsy bulk tissue fractionation process was investigated on the cellular level using a custom-built histotripsy transducer incorporated into the stage of an inverted microscope, which was coupled to high-speed imaging. Histotripsy was applied to optically transparent tissue phantoms with an embedded layer of breast cancer cells to mimic real tissue with cells adherent to a three dimensional matrix, and the bubble-cell interactions produced by a single histotripsy pulse as well as over multiple histotripsy pulses were imaged. This study represents the first time the histotripsy tissue fractionation process has been visualized on the cellular level in a relevant tissue-mimicking environment, with results supporting the hypothesis that the rapid expansion and collapse of histotripsy bubbles fractionate tissue by inducing large transient strains on the adjacent tissue structures. These large transient strains result in the creation of precise lesions matching the bubble cloud size, shape, and location, with sharp boundaries between the fractionated lesion and the intact surrounding tissue.

To experimentally investigate the process of tissue fractionation on the cellular level in a relevant tissue-mimicking environment, breast cancer cells embedded inside a tissue mimicking phantom were exposed to histotripsy pulses applied by a $2 \mathrm{MHz}$ transducer. Results from the single pulse experiments demonstrated bubbles induced cell displacement/deformation to the surrounding cells during both expansion and collapse, with the largest cell displacements occurring after bubble collapse to the cells immediately adjacent to the bubble. Similar results were seen for the multi-pulse experiments, with results showing rupture/removal of all cells directly exposed to the histotripsy bubble cloud. Over the course of multiple pulses, histotripsy incrementally fractionated the cells at the focus, forming a sharp lesion matching previous histological observations (Parsons et al. 2006a; Roberts et al. 2006; Hall et al. 2007; 
Vlaisavljevich et al. 2013b). This cell fractionation and lesion formation was limited to the region exposed to the histotripsy bubbles, with cells further away from the bubbles (i.e. > 25-50 $\mu \mathrm{m})$ remaining intact. This observation shows the precision of histotripsy in creating lesions with sharply demarcated boundaries that will remain localized even if histotripsy is applied beyond the point of complete fractionation. Thus, the mechanical nature of histotripsy ensures damage will only occur in the regions in which bubbles are produced, preventing the risk of collateral damage from overtreatment as is common to other ablation methods such as thermal therapy. These experimental findings were further supported by the single bubble model, which demonstrated that histotripsy exerts large transient strains on the surrounding media, with the largest strains occurring in the regions immediately adjacent to the bubble. Results from the single bubble simulations demonstrated a significant decrease in particle displacement and strain with increasing distance from the bubble following an inverse power relationship, closely matching the findings from the single pulse experiments which showed a significant decrease in cell displacement with increasing distance from the bubble wall. Both the experimental and simulation results support the hypothesis that histotripsy bubbles induce large strains only in the region directly adjacent to the bubbles, helping to explain why histotripsy-induced tissue damage is localized to the regions directly exposed to the bubbles.

Overall, the results from this work support our hypothesis that localized tissue fractionation is caused by the large transient strains induced by the expansion and collapse of the histotripsy bubbles, providing significant insight into the process of forming lesions with sharp boundaries between completely fractionated and intact tissue structures. In addition, the results demonstrate that the cellular response to histotripsy is dependent on the entire tissue microenvironment, with adherent cells being deformed along with the ECM. This observation 
highlights the importance of the tissue phantom used in this work. In preliminary experiments conducted without the top fibrin layer, the vast majority of cells were detached within in a single pulse, including cells farther away from the bubbles. This observation makes sense based on previous work showing that histotripsy is dependent upon tissue mechanical properties (Xu and Bigelow 2011; Vlaisavljevich et al. 2014a; Vlaisavljevich et al. 2014b; Xu et al. 2014; Vlaisavljevich et al. 2015a; Vlaisavljevich et al. 2015b), which are determined by the extracellular matrix, intracellular matrix, and cell-matrix adhesions. During the multi-pulse experiments, cells at the bubble cloud boundary were deformed numerous times and often bisected multiple times before complete removal. These results suggest that histotripsy cell fractionation is better understood by viewing the cells as one component of a tissue network that includes both intracellular and extracellular structures. Cell bisection occurs as this matrix network is broken down, with cells simply being one component of the overall structure that is fractionated. This view of the histotripsy fractionation process helps to explain the previous observation that stiffer tissues are more resistant to histotripsy (Vlaisavljevich et al. 2014a; Vlaisavljevich et al. 2015b), as changes in the stiffness of tissue components (i.e. cells, matrix, adhesions) would impact both the strain induced by the bubbles and the fracture strength of the tissue. Future work will investigate the effects of histotripsy on a variety of cell types in phantoms with a range of ECM stiffness.

Another observation worth noting is that, for all experiments, bubbles were only generated in regions between cells, with no observed instances of cavitation forming inside of a cell. This finding suggests that the cavitation threshold is lower in the extracellular fluid space between cells than it is inside of the cells. Previous work has indicated that histotripsy bubbles are formed in the water inside of the tissue (Maxwell et al. 2013; Vlaisavljevich et al. 2015a). It 
is therefore possible that the cavitation threshold in the extracellular space may be lower than the cavitation threshold in the intracellular space due to a decrease in free water inside the cell, which is densely packed and primarily contains water that is bound to proteins and ions (Clegg 1984; Leterrier 2001; Fels et al. 2009). Future work will investigate this possibility.

\section{Conclusion}

This work represents the first time the histotripsy tissue fractionation process has been viewed at the cellular level in a relevant tissue-mimicking environment, with the results supporting our hypothesis that histotripsy tissue fractionation is caused by the large transient strains applied during both bubble expansion and collapse. Cell deformation was observed during both bubble expansion and collapse, with the largest deformations and cell rupture occurring during collapse for cells immediately adjacent to the bubbles. Both experimental and theoretical results demonstrated a significant decrease in cell displacement with increasing distance from the bubbles, helping to explain the formation of the sharp lesions formed in histotripsy. Over the course of multiple pulses, histotripsy was observed to incrementally fractionate all of the cells in the focal region, forming a precise lesion matching the bubble cloud size, shape, and location, with sharp boundaries between the fractionated lesion and the intact surrounding tissue. Overall, the results of this study provide an understanding of the tissue fractionation process that will help guide the future development of histotripsy.

\section{Acknowledgements}

The authors would like to thank the Takayama Laboratory for supplying the breast cancer cells used in this work. This material is based upon work supported by the National Science 
Foundation Graduate Research Fellowship. This work was supported by grants from National Institute of Biomedical Imaging And Bioengineering (NIBIB) of the National Institutes of Health under Award Number R01EB008998, National Institute of Neurological Disorders and Stroke

(NINDS) of the National Institutes of Health under Award Number R21NS093121, a Research Scholar Grant from the American Cancer Society (RSG-13-101-01-CCE), a National Science Foundation Grant (CBET 1253157), and The Hartwell Foundation. Disclosure notice: Drs. Eli Vlaisavljevich, Charles Cain, and Zhen Xu have financial interests and/or other relationship with HistoSonics Inc.

\section{References}

Barbee KA. Mechanical cell injury. Ann N Y Acad Sci 2005;1066:67-84.

Brujan EA. The role of cavitation microjets in the therapeutic applications of ultrasound. Ultrasound Med Biol 2004;30:381-7.

Chen H, Kreider W, Brayman AA, Bailey MR, Matula TJ. Blood vessel deformations on microsecond time scales by ultrasonic cavitation. Phys Rev Lett 2011;106:034301.

Clegg JS. Properties and Metabolism of the Aqueous Cytoplasm and Its Boundaries. American Journal of Physiology 1984;246:R133-R51.

Cochran SA, Prausnitz MR. Sonoluminescence as an indicator of cell membrane disruption by acoustic cavitation. Ultrasound Med Biol 2001;27:841-50.

Fan ZZ, Liu HY, Mayer M, Deng CX. Spatiotemporally controlled single cell sonoporation. Proceedings of the National Academy of Sciences of the United States of America 2012;109:16486-91.

Fels J, Orlov SN, Grygorczyk R. The hydrogel nature of mammalian cytoplasm contributes to osmosensing and extracellular pH sensing. Biophys J 2009;96:4276-85.

Gaudron R, Warnez MT, Johnsen E. Bubble dynamics in a viscoelastic medium with nonlinear elasticity. Journal of Fluid Mechanics 2015;766:

Hall TL, Kieran K, Ives K, Fowlkes JB, Cain CA, Roberts WW. Histotripsy of rabbit renal tissue in vivo: temporal histologic trends. J Endourol 2007;21:1159-66.

Hammond NA, Kamm RD. Elastic deformation and failure in protein filament bundles: Atomistic simulations and coarse-grained modeling. Biomaterials 2008;29:3152-60.

Hempel CR, Hall TL, Cain CA, Fowlkes JB, Xu Z, Roberts WW. Histotripsy fractionation of prostate tissue: local effects and systemic response in a canine model. J Urol 2011;185:1484-9.

Holzapfel GA. Nonlinear solid mechanics : a continuum approach for engineering. Chichester ; New York: Wiley, 2000.

Kilinc D, Gallo G, Barbee KA. Mechanical membrane injury induces axonal beading through localized activation of calpain. Exp Neurol 2009;219:553-61. 
Kimmel E. Cavitation bioeffects. Crit Rev Biomed Eng 2006;34:105-61.

Kotlarchyk MA, Shreim SG, Alvarez-Elizondo MB, Estrada LC, Singh R, Valdevit L, Kniazeva E, Gratton E, Putnam AJ, Botvinick EL. Concentration independent modulation of local micromechanics in a fibrin gel. PLoS One 2011;6:e20201.

Leterrier JF. Water and the cytoskeleton. Cell Mol Biol (Noisy-le-grand) 2001;47:901-23.

Li ZG, Liu AQ, Klaseboer E, Zhang JB, Ohl CD. Single cell membrane poration by bubbleinduced microjets in a microfluidic chip dagger. Lab on a Chip 2013;13:1144-50.

Lin KW, Kim Y, Maxwell AD, Wang TY, Hall TL, Xu Z, Fowlkes JB, Cain CA. Histotripsy beyond the intrinsic cavitation threshold using very short ultrasound pulses: microtripsy. IEEE Trans Ultrason Ferroelectr Freq Control 2014;61:251-65.

Maxwell AD, Cain CA, Hall TL, Fowlkes JB, Xu Z. Probability of cavitation for single ultrasound pulses applied to tissues and tissue-mimicking materials. Ultrasound Med Biol 2013;39:449-65.

Maxwell AD, Wang TY, Cain CA, Fowlkes JB, Sapozhnikov OA, Bailey MR, Xu Z. Cavitation clouds created by shock scattering from bubbles during histotripsy. J Acoust Soc Am 2011;130:1888-98.

Maxwell AD, Wang TY, Yuan L, Duryea AP, Xu Z, Cain CA. A tissue phantom for visualization and measurement of ultrasound-induced cavitation damage. Ultrasound Med Biol 2010;36:2132-43.

Ofek G, Dowling EP, Raphael RM, McGarry JP, Athanasiou KA. Biomechanics of single chondrocytes under direct shear. Biomech Model Mechanobiol 2010;9:153-62.

Ohl CD, Arora M, Ikink R, de Jong N, Versluis M, Delius M, Lohse D. Sonoporation from jetting cavitation bubbles. Biophysical Journal 2006;91:4285-95.

Owens GE, Miller RM, Ensing G, Ives K, Gordon D, Ludomirsky A, Xu Z. Therapeutic ultrasound to noninvasively create intracardiac communications in an intact animal model. Catheter Cardiovasc Interv 2011;77:580-8.

Parsons JE, Cain CA, Abrams GD, Fowlkes JB. Pulsed cavitational ultrasound therapy for controlled tissue homogenization. Ultrasound Med Biol 2006a;32:115-29.

Parsons JE, Cain CA, Fowlkes JB. Cost-effective assembly of a basic fiber-optic hydrophone for measurement of high-amplitude therapeutic ultrasound fields. J Acoust Soc Am 2006b;119:1432-40.

Quinto-Su PA, Kuss C, Preiser PR, Ohl CD. Red blood cell rheology using single controlled laser-induced cavitation bubbles. Lab on a Chip 2011;11:672-8.

Roberts WW, Hall TL, Ives K, Wolf JS, Jr., Fowlkes JB, Cain CA. Pulsed cavitational ultrasound: a noninvasive technology for controlled tissue ablation (histotripsy) in the rabbit kidney. J Urol 2006;175:734-8.

Sankin GN, Yuan F, Zhong P. Pulsating Tandem Microbubble for Localized and Directional Single-Cell Membrane Poration. Physical Review Letters 2010;105:

Sundaram J, Mellein BR, Mitragotri S. An experimental and theoretical analysis of ultrasoundinduced permeabilization of cell membranes. Biophys J 2003;84:3087-101.

Vlaisavljevich E, Durmaz YY, Maxwell A, Elsayed M, Xu Z. Nanodroplet-mediated histotripsy for image-guided targeted ultrasound cell ablation. Theranostics 2013a;3:851-64.

Vlaisavljevich E, Kim Y, Allen S, Owens G, Pelletier S, Cain C, Ives K, Xu Z. Image-guided non-invasive ultrasound liver ablation using histotripsy: feasibility study in an in vivo porcine model. Ultrasound Med Biol 2013b;39:1398-409. 
Vlaisavljevich E, Kim Y, Owens G, Roberts W, Cain C, Xu Z. Effects of tissue mechanical properties on susceptibility to histotripsy-induced tissue damage. Phys Med Biol 2014a;59:253-70.

Vlaisavljevich E, Lin KW, Maxwell A, Warnez M, Mancia L, Singh R, Putnam A, fowlkes JB, Johnsen E, Cain C, Xu Z. Effects of Ultrasound Frequency and Tissue Stiffness on the Histotripsy Intrinsic Threshold for Cavitation. Ultrasound Med Biol 2015a;

Vlaisavljevich E, Lin KW, Warnez M, Singh R, Mancia L, Putnam A, Johnsen E, Cain C, Xu Z. Effects of Tissue Stiffness, Ultrasound Frequency, and Pressure on Histotripsy-induced Cavitation Bubble Behavior. Phys Med Biol 2015b;

Vlaisavljevich E, Maxwell A, Warnez M, Johnsen E, Cain CA, Xu Z. Histotripsy-induced cavitation cloud initiation thresholds in tissues of different mechanical properties. IEEE Trans Ultrason Ferroelectr Freq Control 2014b;61:341-52.

Vogel A, Busch S, Parlitz U. Shock wave emission and cavitation bubble generation by picosecond and nanosecond optical breakdown in water. Journal of the Acoustical Society of America 1996;100:148-65.

Warnez MT, Johnsen E. Numerical modeling of bubble dynamics in viscoelastic media with relaxation. Physics of Fluids 2015;27:

Wu J. Shear stress in cells generated by ultrasound. Prog Biophys Mol Biol 2007;93:363-73.

$\mathrm{Xu}$ J, Bigelow TA. Experimental investigation of the effect of stiffness, exposure time and scan direction on the dimension of ultrasound histotripsy lesions. Ultrasound Med Biol 2011;37:1865-73.

Xu J, Bigelow TA, Davis G, Avendano A, Shrotriya P, Bergler K, Hu Z. Dependence of ablative ability of high-intensity focused ultrasound cavitation-based histotripsy on mechanical properties of agar. J Acoust Soc Am 2014;136:3018.

$\mathrm{Xu} \mathrm{Z}$, Fowlkes JB, Ludomirsky A, Cain CA. Investigation of intensity thresholds for ultrasound tissue erosion. Ultrasound Med Biol 2005a;31:1673-82.

$\mathrm{Xu}$ Z, Fowlkes JB, Rothman ED, Levin AM, Cain CA. Controlled ultrasound tissue erosion: the role of dynamic interaction between insonation and microbubble activity. J Acoust Soc Am 2005b; 117:424-35.

Xu Z, Owens G, Gordon D, Cain C, Ludomirsky A. Noninvasive creation of an atrial septal defect by histotripsy in a canine model. Circulation 2010;121:742-9.

Yuan F, Yang C, Zhong P. Cell membrane deformation and bioeffects produced by tandem bubble-induced jetting flow. Proceedings of the National Academy of Sciences of the United States of America 2015; 


\section{Figure Legends.}

Figure 1. (A) Experimental set-up showing custom-built microscope transducer-tank. (B) Example acoustic waveform. (C) Schematic of tissue phantom containing a layer of breast cancer cells inside a fibrin gel.

Figure 2. Radial and Polar Strain Schematic. Schematic show the directions of the radial $\left(E_{r r}\right)$ and polar $\left(E_{\theta \theta}\right)$ strains calculated by the single bubble simulation during (A) bubble expansion and (B) bubble collapse.

Figure 3. Cancer cells exposed to a single histotripsy pulse were significantly deformed during both bubble expansion and collapse, with the largest deformations observed during collapse (between $46 \mu \mathrm{s}$ and $74.75 \mu \mathrm{s}$ ). Arrows indicate regions containing bubbles (B) and the deformed cells (DC).

Figure 4. Cell displacement during bubble collapse. Plot shows the cell displacement observed during bubble collapse as a function of distance from the bubble wall. Results demonstrated a significant decrease in displacement for cells farther away from the bubble wall.

Figure 5. Cancer cells exposed to 1000 histotripsy pulses were repeatedly deformed by bubbles (B) over multiple pulses until cell rupture/removal was achieved, forming a well-defined lesion (L) in the focal zone matching lesions that have previously been observed for histological analysis of histotripsy lesions. 
Figure 6. Cancer cells at the boundary of the histotripsy lesion were repeatedly deformed (pulled towards bubble) and often bisected multiple times before complete removal. Arrow indicates a breast cancer cell bisected over multiple pulses.

Figure 7. Bubble-induced strain simulation. Color plots show the magnitudes of (A) radial and (B) polar strains produced during the expansion of a single histotripsy bubble. To more clearly contrast strain magnitudes, the absolute value of the radial strains is plotted. Black lines show the trajectories, $\mathrm{r}(\mathrm{t})$ of particles starting at various distances, $r^{*}$ from the bubble nucleus. Plots of the magnitudes of (C) radial and (D) polar strains experienced by particles moving along these trajectories demonstrate a significant decrease in strain with increasing distance from the bubble.

Figure 8. Bubble simulation: Maximum Strain vs. Distance. Plots show the maximum (A) radial and (B) polar strains produced during the expansion of a single bubble for points located 0 $\mu \mathrm{m}-500 \mu \mathrm{m}$ from the initial nucleus of a bubble expanded to a $R_{\max }$ of $\sim 200 \mu \mathrm{m}$. Results demonstrated an exponential decrease in maximum strain with increasing distance from the bubble. 

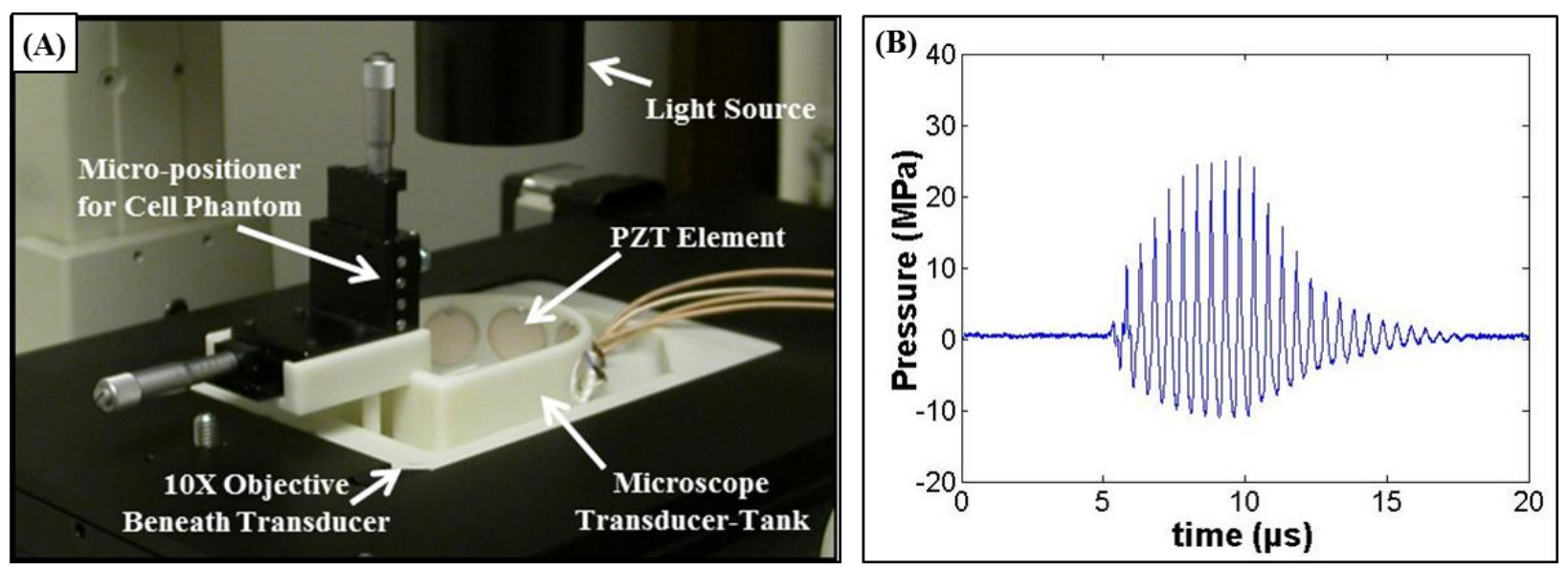

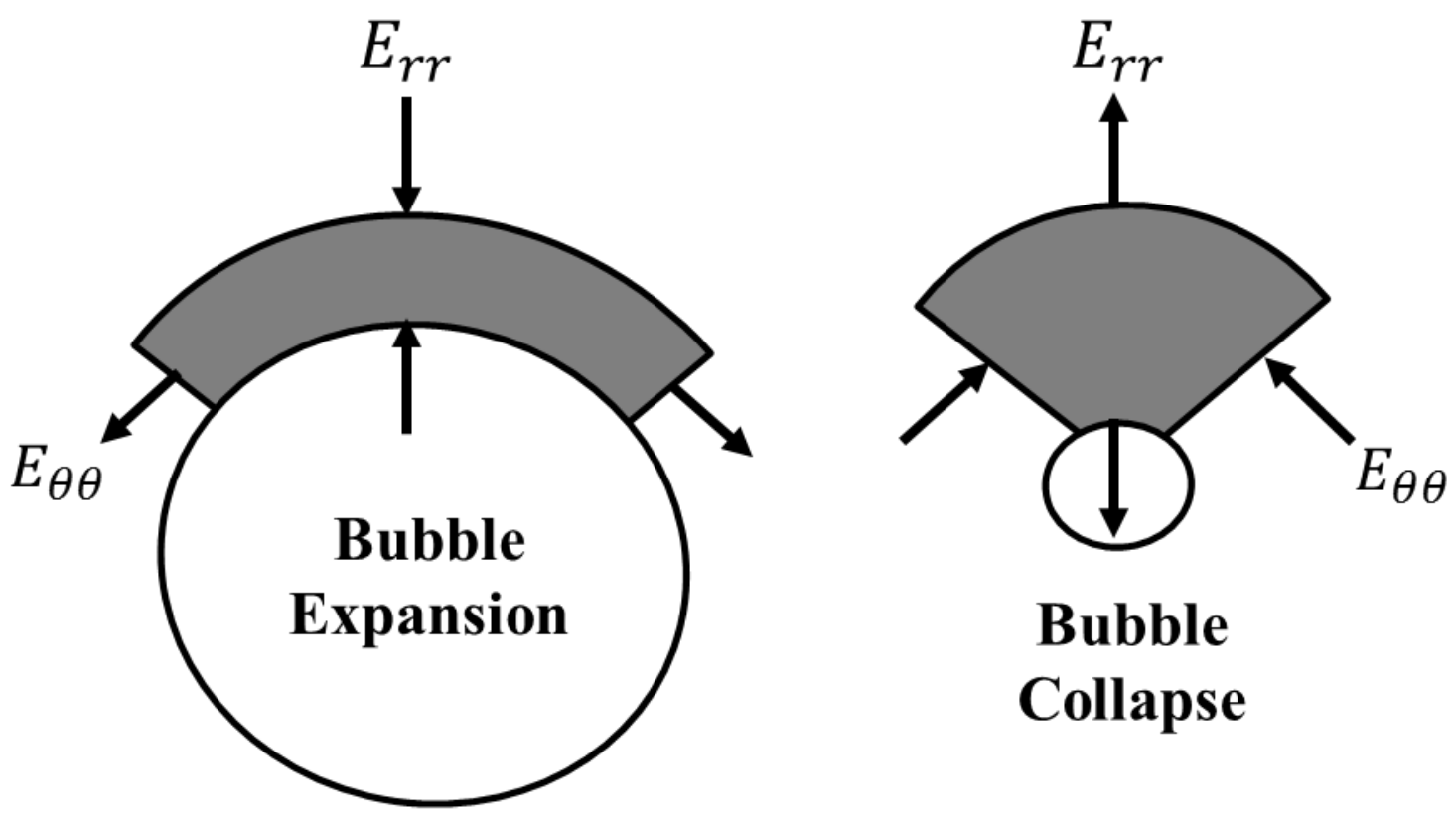

(A)

(B) 


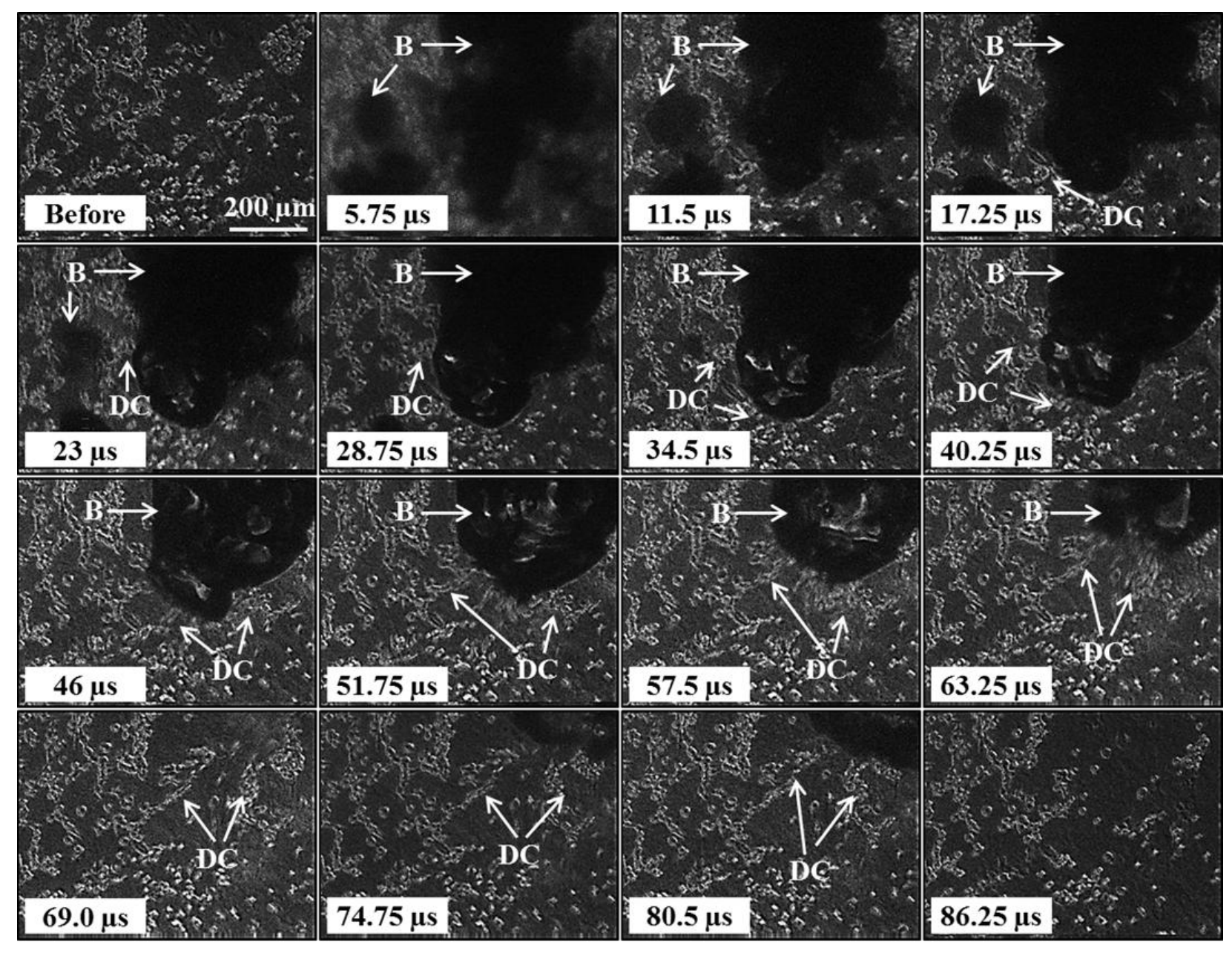




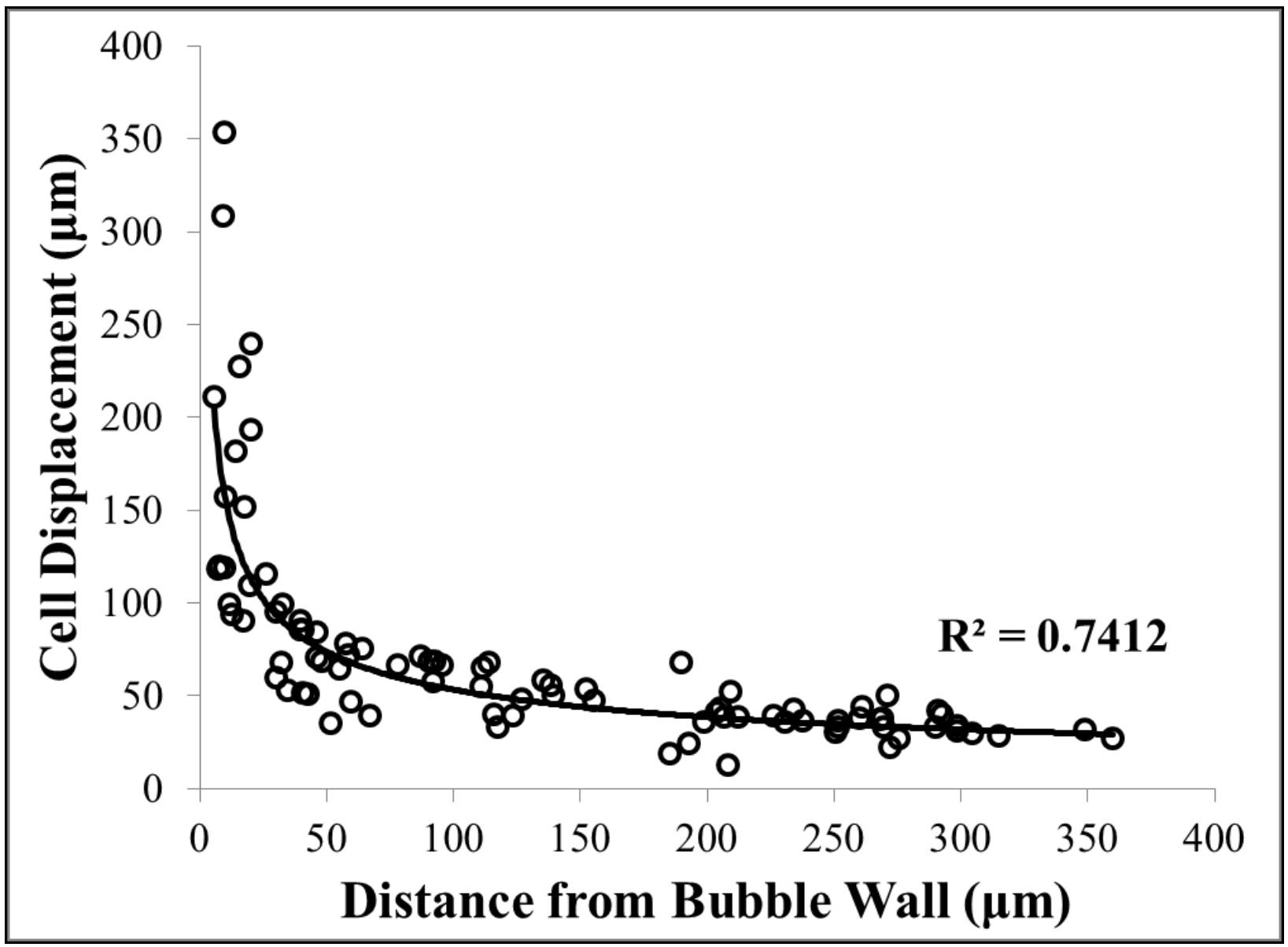



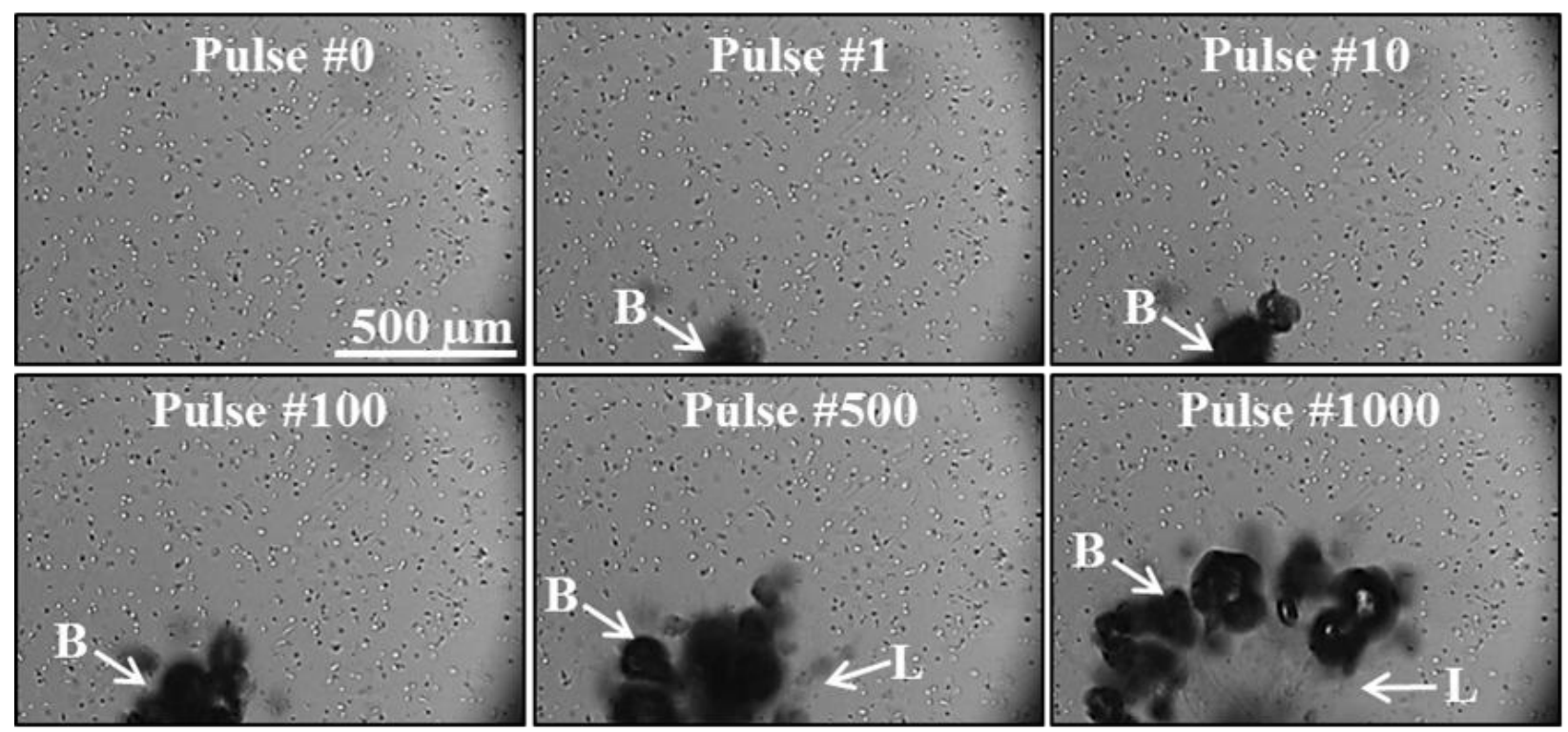


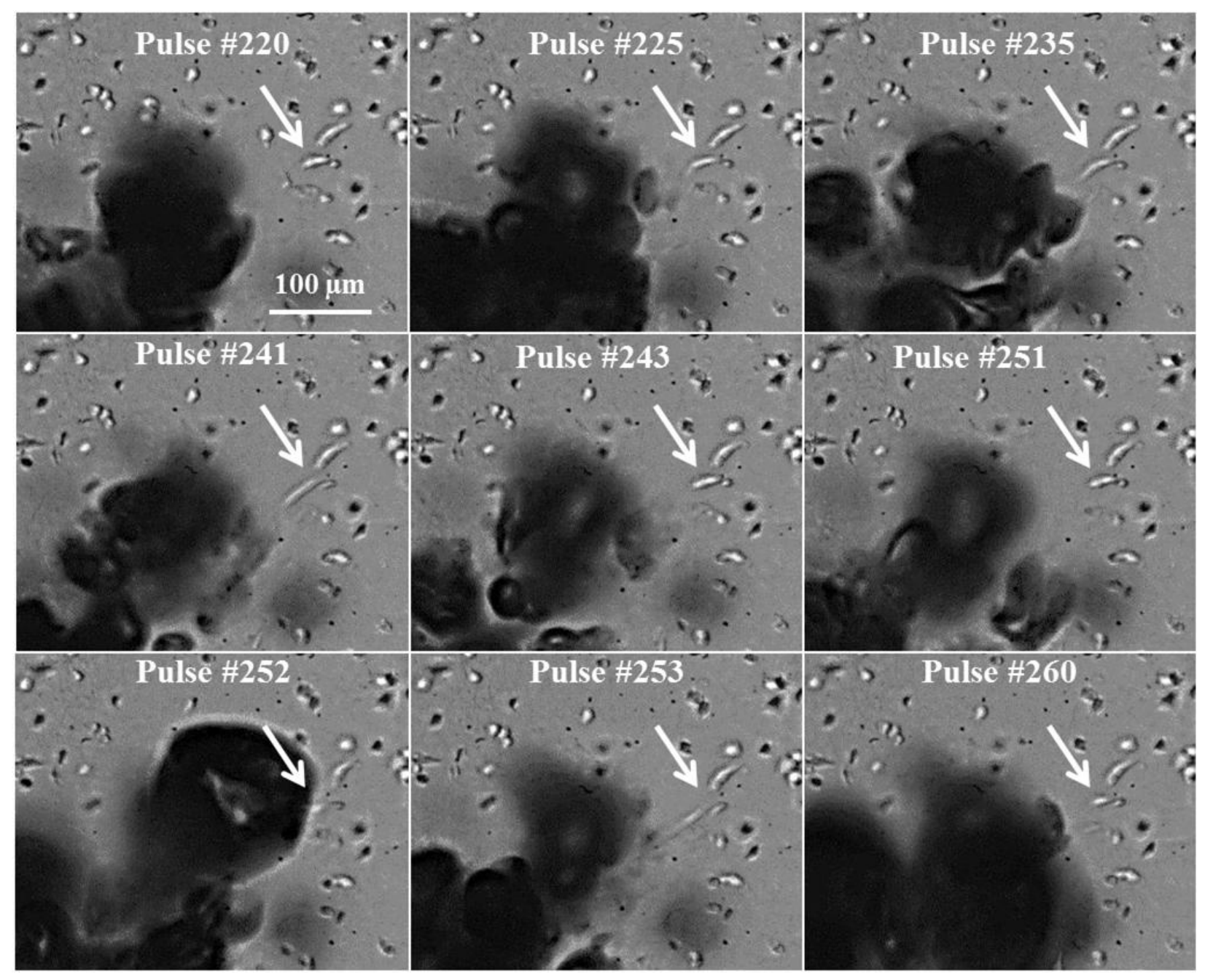


(A) $\quad\left|E_{\pi T}\right|(\mu m / \mu m)$

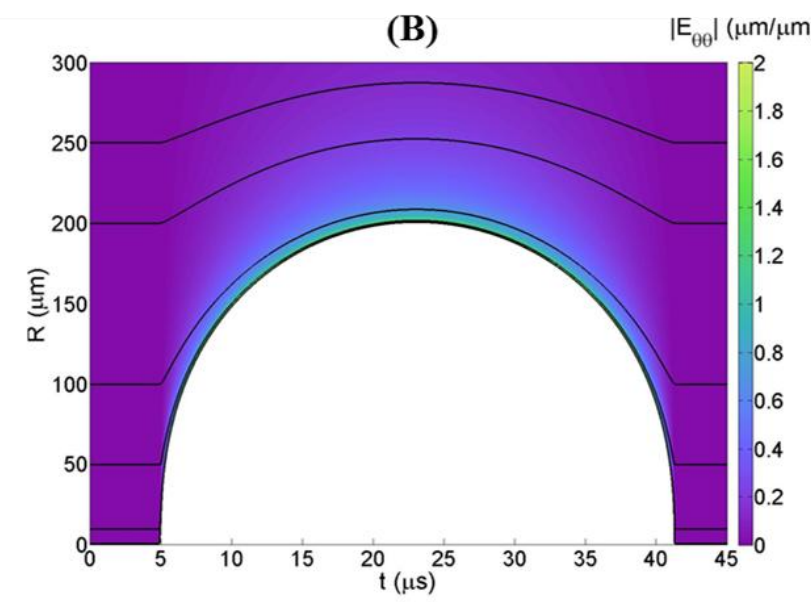

(C)

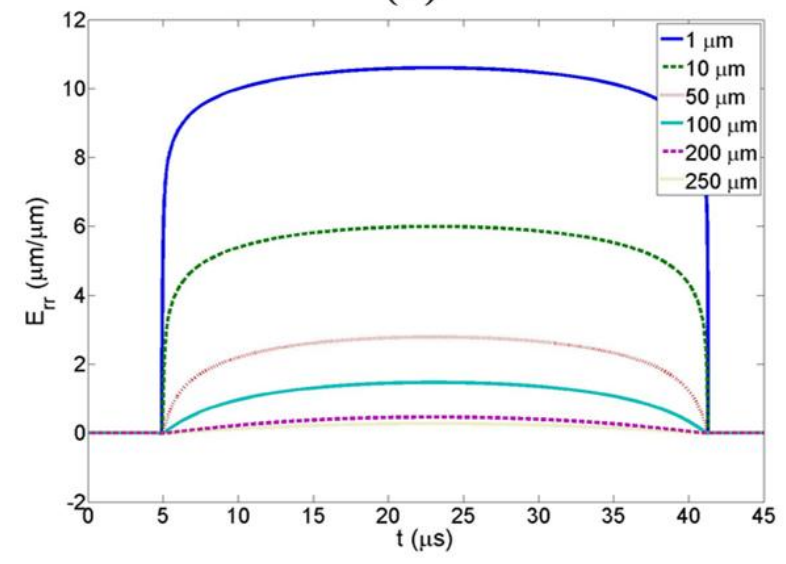

(D)

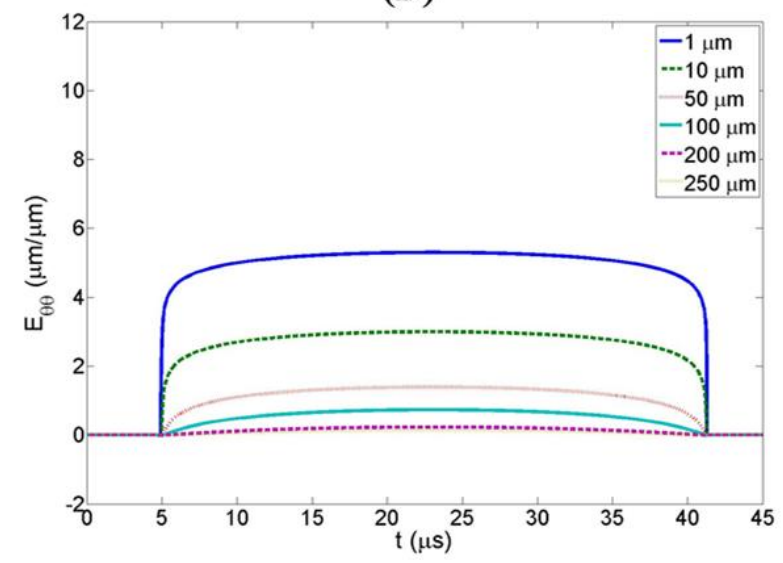


(A)

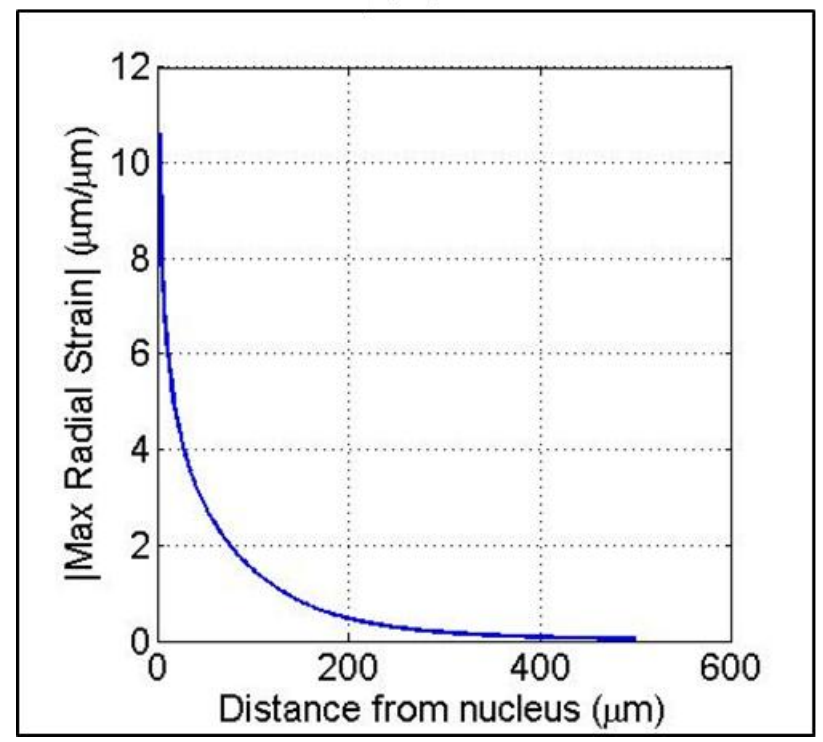

(B)

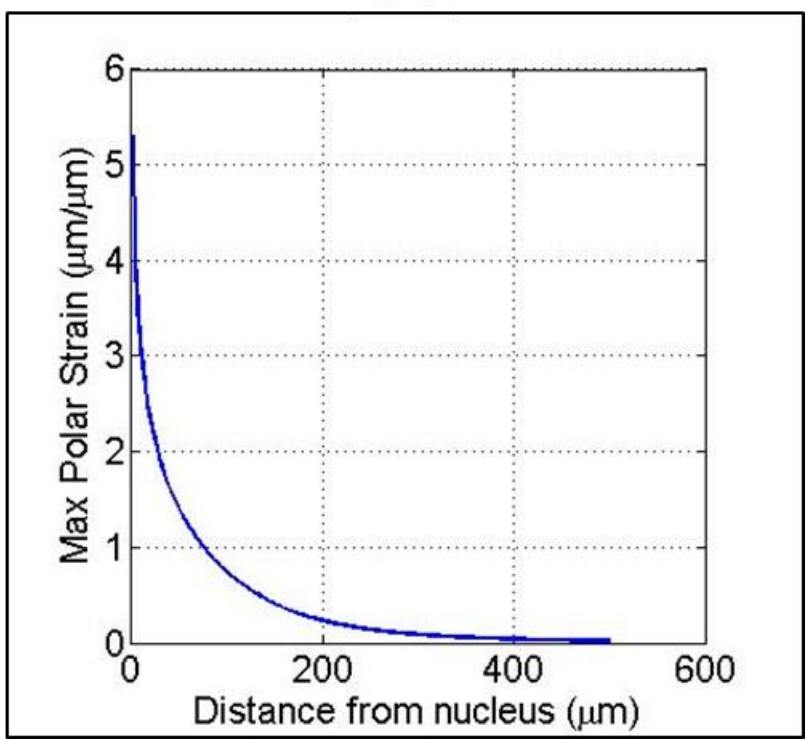

